\title{
Effects of medium variation and fermentation time on the antioxidant and antimicrobial properties of Kombucha
}

\author{
Batul Moiz Vohra, Shazrul Fazry, Fareed Sairi, Othman Babul-Airianah* \\ Faculty of Science and Technology, Universiti Kebangsaan Malaysia, 43600, Bangi, Selangor \\ * Corresponding author: airianah@ukm.edu.my
}

\section{Article history}

Received 28 December 2018

Revised 22 February 2019

Accepted 4 April 2019

Published Online 15 May 2019

\begin{abstract}
Kombucha is a traditional fermented drink and has recently gained popularity due to its numerous claims on therapeutic effects. It is prepared by sweetening black tea and fermenting using a symbiotic culture of acetic acid bacteria and yeasts known as SCOBY. The drink can also be brewed using different types of tea and carbon sources. An investigation was conducted to characterize the antioxidant and antimicrobial properties of kombucha by using variation of carbon sources: white sugar $(\mathrm{S})$, jaggery $(\mathrm{J})$ and Kelulut honey $(\mathrm{H})$ in black tea $(B T)$ and green tea $(\mathrm{GT})$ media over a period of 7 , 14,28 and 60 days. The antioxidant and antimicrobial efficacies were tested post fermentation. All samples shown the highest value of 2,2-diphenyl-1-picrylhydrazyl (DPPH) inhibition for antioxidant activity at 7 days of fermentation but subsequent decrease was observed with longer fermentation time except for the combination of green tea with jaggery $(G T+J)$. Fermentation of green tea with white sugar $(G T+S)$ showed the highest value with $84 \%$. On the other hand, black tea with jaggery $(B T+J)$ showed least DPPH activity of $9 \%$. Fermentation with green tea demonstrated a higher antioxidant activity compared to black tea, whereas fermentation of any tea with jaggery showed the least antioxidant activity in comparison to white sugar and honey. Thus, it could be said that extended periods of fermentation would reduce the DPPH inhibition of kombucha and jaggery itself as carbon source showed an interesting property. Antimicrobial activity was tested using disk diffusion method against $E$. coli, $S$. aureus, $P$. aeruginosa, $B$. subtilis and $S$. marcescens. The efficacy was increased with time of fermentation for all combinations. Combination of black tea with all carbon sources was found to exhibit good antimicrobial activity.
\end{abstract}

Keywords: kombucha, SCOBY, fermented tea, antioxidant, antimicrobial

(C) 2019 Penerbit UTM Press. All rights reserved

\section{INTRODUCTION}

Kombucha is a popular beverage amongst the traditional fermented foods and has gained popularity across the. It is a beverage prepared by fermenting sugared tea with a symbiotic colony of acetic acid bacteria (AAB) and yeasts, commonly called SCOBY. It is extensively consumed in the world for its prophylactic and therapeutic properties. It is believed to be therapeutic against cardiovascular diseases, AIDS, diabetes, high blood pressure, digestive ailments on account of its health promoting metabolites and high concentrations of acids, ethanol and carbon dioxide (Sreeramulu et al., 2000; Dufresne and Farnworth, 2000; Vīna et al., 2014).

There are two distinct portions of kombucha: the cellulosic and the tea or broth portion. The cellulosic portion is made of a cellulose synthesized by the bacteria and yeasts of the SCOBY whereas the tea portion is the fermented liquid which is consumed by the brewer (Greenwalt et al., 2000). The major strains of bacteria found are Acetobacter xylinum, Acetobacter xylinoides, Bacterium gluconicum and the yeasts include Schizosaccharomyces pombe, Saccharomyces ludwigii, Zygosaccharomyces rouxii, Pichia membranaefaciens (Chen and Liu, 2000; Hesseltine, 1965). Sucrose is the main and traditional carbon source used in the making of kombucha. The yeasts in the SCOBY will hydrolyze it to glucose and fructose using invertase and ferment it to ethanol before being oxidized to acetic acid by the acetic acid bacteria (Velićanski et al., 2014; Sievers et al., 1995). This is the main metabolic pathway of kombucha.

Kombucha is also reported to be an antioxidant, where green tea as a substrate is proven to be a better antioxidant in comparison to black tea (Fu et al., 2014). Its health benefits are said to come from antioxidant nature of tea. Antioxidants can neutralize the free radicals produced in our body by donating an electron. Free radicals can cause oxidative stress; it is the state when the body is overwhelmed with free radicals and the antioxidant defense is low. Besides than non-fermented tea, kombucha also has phenolic content which is responsible for the antioxidant efficacy (Yang et al., 2008; Vīna et al., 2014). Antioxidants can help in prevention of autoimmune diseases, Alzheimer's, rheumatism, metabolic disorders, asthma and kidney dysfunction (Dufrense and Fanworth, 2000). DPPH assay is conducted to test the antioxidant effect. The DPPH scavenging ability of the sample is the basis of this assay. The electron donating capacity of the samples is tested by bleaching the purple reagent of DPPH into yellow color (Sharma and Bhatt, 2009).

Kombucha has also shown antimicrobial properties. Greenwalt et al. (1998) attributed the antimicrobial activity of kombucha to its acetic acid content. Sreeramulu et al. (2000), however, concluded that the antimicrobial activity of kombucha was due to the presence of certain molecules in kombucha post fermentation. According to Bhattacharya et al. (2016), kombucha exerted antimicrobial activity due to the 
presence of catechins in the tea and also a compound isorhamnetin. It showed potent antimicrobial efficacy after 14th day of fermentation.

As kombucha is gaining more popularity nowadays, the media and carbon source used are experiencing the changes by brewers. The time of fermentation is also varied by different brewers. Different carbon sources may react differently with the microbial flora of the tea and therefore, it can be hypothesized that the therapeutic efficacy can vary with the change in media and fermentation time. However, to what extent they may vary is not known. To the best of our knowledge, no study has dealt with the investigation on variation in therapeutic efficacies with changes in media and fermentation time.

This investigation was aimed to determine the effects of medium variation and fermentation time on kombucha's antioxidant and antimicrobial activities. In order to accomplish the aim of this study two types of teas; black tea and green tea were mixed with different carbon sources; white sugar, Kelulut honey and jaggery. Different fermentation periods were studied to determine and understand the effects of the substrate and time of fermentation towards the therapeutic activity.

\section{EXPERIMENTAL}

\section{Preparation of Kombucha Tea samples}

Samples were prepared using one tea (black or green tea) and one carbon source (white sugar, jaggery or Kelulut honey). The tea bags, black tea (Lipton brand) and green tea (OSK brand) were obtained from the local market. Carbon sources such as white sugar (CSR brand) and jaggery (Bintang brand) were obtained from the local market while the Kelulut honey was obtained from Malaysian Genome Institute (MGI). Various combinations of samples such as black tea with white sugar $(\mathrm{BT}+\mathrm{S})$, black tea with Kelulut honey $(\mathrm{BT}+\mathrm{H})$, black tea with jaggery $(\mathrm{BT}+\mathrm{J})$, green tea with white sugar $(\mathrm{GT}+\mathrm{S})$, green tea with Kelulut honey $(\mathrm{GT}+\mathrm{H})$ and green tea with jaggery $(\mathrm{GT}+\mathrm{J})$ were prepared. Samples with only tea but without carbon sources; green tea only (GTO) and black tea only (BT-O) were also used for comparison. Similarly, samples with no tea but only one carbon source; white sugar only (S$\mathrm{O})$, Kelulut honey only $(\mathrm{H}-\mathrm{O})$ and jaggery only $(\mathrm{J}-\mathrm{O})$ were also used for comparison. Briefly, tea at $2 \%(\mathrm{w} / \mathrm{v})$ was boiled together with $10 \%$ of carbon source and steeped for 10 mins. This mixture was then allowed to cool down to room temperature. The tea (green tea or black tea) was filled in sterile falcon tubes and $10 \%(\mathrm{v} / \mathrm{v})$ old kombucha was added as inoculum for fermentation. The final volume of all samples was $40 \mathrm{~mL}$. All samples were covered with cheesecloth and kept at room temperature for fermentation for 7, 14, 28 and 60 days. The samples were prepared in triplicates for each time point. Before proceeding with DPPH assay and antimicrobial test, all samples were collected and filtered using a 0.2 millipore syringe filter to make sure that no debris was remained in them. Non-fermented tea and carbon sources were used as negative control (NC).

\section{Determination of antioxidant activity}

Antioxidant activity of the samples was tested by DPPH $(2,2-$ diphenyl-1-picrylhydrazyl) assay. The reagent was prepared by dissolving $4 \mathrm{mg}$ of the reagent powder in $100 \mathrm{~mL}$ methanol. A total of $100 \mu \mathrm{L}$ sample and reagent were pipetted into a microplate and incubated for 30 mins in the dark at room temperature. The plates were read at $517 \mathrm{~nm}$ using a microplate reader (BioTek Epoch). DPPH inhibition was calculated using the formula (1)

$$
\frac{\left(A_{0}-A_{i}\right)}{A_{0}} \times 100
$$

where $\mathrm{A}_{0}=$ Absorbance of blank, $\mathrm{A}_{\mathrm{i}}=$ Absorbance of sample. Ascorbic Acid was used as a standard and distilled water was used as the blank. Non-fermented tea was served as control. The assay was performed in triplicates and standard deviation was calculated.

\section{Determination of antimicrobial activity}

Antimicrobial effect was conducted via disk diffusion method according to the method followed by Greenwalt et al. (1998) with few modifications against $E$. coli, $P$. aeruginosa, B. subtilis, $S$. aureus and
S. marcescens. Briefly, a single colony of each bacterium was inoculated in $5 \mathrm{~mL}$ of nutrient broth media and incubated for 24 hours at $30^{\circ} \mathrm{C}$. These bacteria were chosen as they are common human pathogens. A total of $0.1 \mathrm{~mL}$ broth from each culture was spreaded evenly on nutrient agar (Sigma Aldrich, Germany) using a spread plating rod. Whattmans filter paper was used to prepare $0.5 \mathrm{~mm}$ disks. The disks were then saturated with the kombucha samples and inoculated on the plates with the lawn of the pathogen with sterile forceps. The plates were incubated for 24 hours at $30^{\circ} \mathrm{C}$. The zone of inhibition was calculated post incubation.

\section{Statistical analysis}

Statistical analysis was performed to determine statistical significance of the mean values of the data obtained using Minitab software version 17. Two-way ANOVA was used to study the variance in the data obtained. According to the ANOVA model, for null hypothesis, it was assumed that the data has no variance and was statistically insignificant whereas an alternative hypothesis was assumed that the mean values of the data were significantly different. Confidence interval of $95 \%$ was used to calculate $\mathrm{F}$ and $\mathrm{P}$ values. The significance was calculated using F-Values and P-Values. With F values $(>>1)$ and $P$-values $(<0.05)$, null hypothesis could be rejected and thus, it could be concluded that the results were statistically significant.

\section{RESULTS AND DISCUSSION}

\section{Antioxidant activity}

The aim was to investigate the influence of media and fermentation time on kombucha's antioxidant properties. The standard positive control taken was ascorbic acid, in which it showed antioxidant activity at $82 \%$ (not shown in Fig. 1). As depicted in Fig.1, all fermented samples studied at different times of fermentation and different media showed antioxidant activity. It was generally believed that polyphenols and catechins in the tea were responsible for the antioxidant properties of kombucha as they could easily give a hydroxyl hydrogen due to resonance stabilization (Fessenden and Fessenden, 1994; Jayabalan et al., 2008)

The highest antioxidant activity was shown at 7 days of fermentation with a decreasing trend over longer fermentation time except for the combination of GT+J. The highest inhibition values for fermented samples were just slightly lower (for fermented black tea) or slightly higher (for fermented green tea) when compared to the nonfermented tea $(\mathrm{NC})$ except for $\mathrm{BT}+\mathrm{J}$ and $\mathrm{GT}+\mathrm{J}$. This showed that kombucha fermentation did not help much in increasing antioxidant properties of the teas, especially black tea. Upon fermentation, the concentrations of epicatechin (EC), epigallocatechin gallate (EGCG), and allocatechin gallate (GCG) were increased which could effectively neutralize the free radicals (Lobo et al., 2017). The increase or decrease of the polyphenols in kombucha was said to be due to the biotransformation of already presented phytochemicals into polyphenols by the enzymes secreted by the SCOBY consortium (Jayabalan et al., 2006). With respect to the negative controls of unfermented tea of samples BT+J and GT+J, fermentation of both teas with jaggery $(\mathrm{BT}+\mathrm{J}$ and $\mathrm{GT}+\mathrm{J})$ would promote the antioxidant activity of the samples. Amongst the combinations studied, the potency of antioxidant was decreased as in following order: $\mathrm{GT}+\mathrm{S}>\mathrm{GT}+\mathrm{H}>$ $\mathrm{BT}+\mathrm{S}>\mathrm{BT}+\mathrm{H}>\mathrm{GT}+\mathrm{J}>\mathrm{BT}+\mathrm{J}$. The best combination thus, was $\mathrm{GT}+\mathrm{S}$

The fermentations with only tea and only sweeteners were also studied to determine the effect of fermentation on tea without the influence of carbon sources and vice versa. Even without the carbon source, green tea was a more potent antioxidant substance as GT-O as it showed $76 \%$ DPPH inhibition for 7th-day sample while BT-O only exhibited DPPH inhibition at $66 \%$. Again, fermented black tea with or without the carbon sources showed lower antioxidant activity when compared to its non-fermented control (NC), in which it was similar to the observation seen for fermented BT-S and BT-H. The antioxidant effect of green tea was higher due to the fact that it has more polyphenols than black tea. Green tea has $30-40 \%$ of extractable polyphenols whereas black tea has 3-10\% (Fatima et al., 2016). Tea 
polyphenols as antioxidants were effective in removing free radicals by playing a preventive role in the oxidative changes of the substrate (Gramza-Michalowska et al., 2016).

In case of samples with only carbon source with no tea, fermented samples of carbon sources ( $\mathrm{S}-\mathrm{O}, \mathrm{H}-\mathrm{O}$ and $\mathrm{J}-\mathrm{O}$ ) showed better antioxidant properties at least after a shorter period of fermentation when compared to their non-fermented negative control. Fermented sample of $\mathrm{J}-\mathrm{O}$ showed better antioxidant activity compared to its combination with black tea $(\mathrm{BT}+\mathrm{J})$. Although both the teas and jaggery by themselves were antioxidant by nature, on mixing them the effect was lost. To the best of our knowledge, no study has reported this observation and thus, further investigation was needed to understand the effect of jaggery on kombucha properties. Overall, the samples have shown a variation in the antioxidant property due to the difference in medium used that affected the fate of the metabolism of the tea (Chu and Chen, 2006).

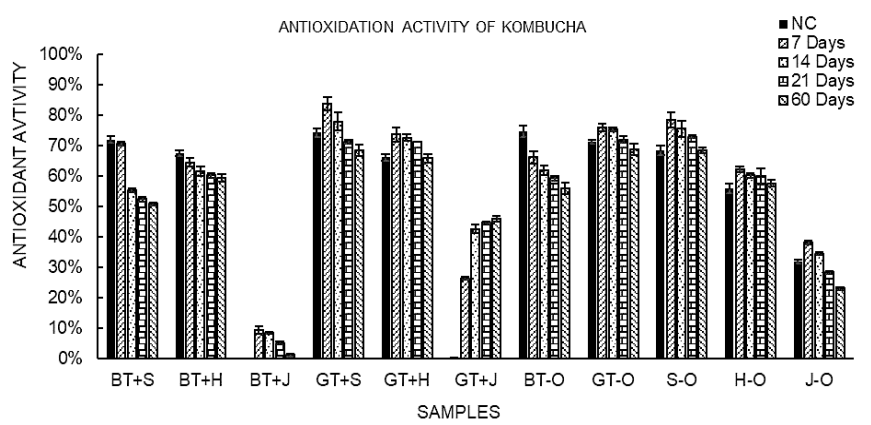

Fig. 2 Graph shows the variation in DPPH inhibition in the samples for fermentation up to 60 days. Error bars represent the standard deviation with $n=3$. Note on terminology: Black tea with white sugar $(B T+S)$, black tea with honey $(B T+H)$, black tea with jaggery $(B T+J)$, green tea with white sugar $(\mathrm{GT}+\mathrm{S})$, green tea with honey $(\mathrm{GT}+\mathrm{H})$, green tea with jaggery $(G T+J)$. black tea only (BT-O), green tea only (GT-O), sugar only (S-O), honey only $(\mathrm{H}-\mathrm{O})$, jaggery only $(\mathrm{J}-\mathrm{O})$ and the non-fermentend sample of each combination as the negative control (NC).

ANOVA was performed to determine if the results were significant and statistically accurate. F-values for the fermentation time and the media (tea and carbon source) were found to be 2923.89 and 187.46, respectively, in which both of them were very high $(\gg>1)$ while the P-values were zero. This implied that null hypothesis of the ANOVA model could be rejected. In other words, the mean of different samples was significantly different from each other. Hence, it could be concluded that fermentation time and media variations have significant effect on the antioxidant properties of the samples.

\section{Antimicrobial Activity}

Kombucha also possessed antimicrobial properties as it has the ability to inhibit the growth of common human pathogens as shown in the Table 1, 2 and 3 (note that all the 7 days fermented kombucha samples did not show any antimicrobial activity, thus data was not included). The fermented samples showed an increase in potency over increase in fermentation time. Only BT $+\mathrm{S}, \mathrm{BT}+\mathrm{H}, \mathrm{BT}+\mathrm{J}$ and $\mathrm{GT}+\mathrm{J}$ of 14 days fermentation showed inhibition zone with $\mathrm{BT}+\mathrm{S}$ was observed to inhibit 3 out of the 5 strains, as summarized in Table 1 . The 28 days samples showed the increase in activity with 10 out of 11 samples by showing antimicrobial activity against the pathogens, as shown in Table 2. Table 3 shows the activity for 60 days fermentation, it could be seen that the samples showed maximum activity and all samples could inhibit almost all the pathogens at different degrees. The negative controls of non-fermented tea and samples of 7 days showed no antimicrobial activity.

Previous study showed that the tea was contained wih catechins such as EC, EGCG, ECG and EGC which attributed to the tea antimicrobial property (Chou et al., 1999). In fermented tea, the fermentation process would produce antimicrobial metabolites such as acids and cause the $\mathrm{pH}$ to drop (Greenwalt et al., 1998; Sreeramulu et al., 2000; Mo et al., 2008). Although green tea possessed more catechins than black tea, it showed less antimicrobial activity, thus proving that fermentation played a crucial role. Various compounds were said to be responsible for the antimicrobial activity. Greenwalt et al. (1999) attributed the acetic acid to be the sole reason for the antibiotic activity in kombucha. Undissociated molecule of the acid could enter in the bacterial cell and the dissociation would take place in the cytoplasm and inhibit the cell growth (Cetojevic-Simin et al., 2008). Study by Bhattacharya et al. (2017) showed that isorhamnetin and catechins in kombucha could impair the growth of the pathogens in a dose dependent manner.

Based on the present study, the fermented black tea with all the carbon sources $(\mathrm{BT}+\mathrm{S}, \mathrm{BT}+\mathrm{H}$ and $\mathrm{BT}+\mathrm{J})$ showed more activity against the pathogen's studies compared to green tea samples. The 60 days samples of BT+S and BT $+\mathrm{J}$ showed antimicrobial activity on all the microbes. Green tea with the carbon sources did not consider as potent. Green tea with sugar $(\mathrm{GT}+\mathrm{S})$ could inhibit $E$. coli and $S$. aureus only after 28th days of fermentation. At the 60 days of fermentation, GT+S would inhibit all the microbes except $P$. aeruginosa. $\mathrm{GT}+\mathrm{J}$ of the 60 days fermentation showed a good activity in inhibiting all the microbes. This combination showed maximum inhibition for B. subtilis. However, in the samples of prolonged fermented tea (Table 3) without the carbon source, green tea (GT-O) was a better antimicrobial agent. It showed high activity against $E$. coli, $S$. marcescens and $P$. aeruginosa.

$\mathrm{BT}+\mathrm{S}$ of 14 days sample could inhibit 3 out of 5 strains and was able to inhibit all 5 by the sample of 60 days while GT+S was started to show inhibition only by the 28 days sample. Since BT+S of 14 days fermentation could inhibit the microbes, it could be said that in comparison to $\mathrm{GT}+\mathrm{S}, \mathrm{BT}+\mathrm{S}$ was a kombucha with better antimicrobial properties. Amongst the different carbon sources studied, H-O and J-O showed inhibition of pathogens on the 28th day whereas samples with S-O showed antimicrobial activity on the 60th day of fermentation only. The fermented sample of J-O showed a better antimicrobial potential in comparison to S-O and H-O. It was able to inhibit E. coli, S. aureus and $S$. marcescens extensively as seen in the Table 2 and 3. J-O was proven to be the most antimicrobial carbon source amongst the ones studied. Thus, it could stand to reason that the presence of carbon source also played a role in the fermentation process in either increasing or decreasing the therapeutic effect.

Table 1 Antimicrobial activity of kombucha samples on $14^{\text {th }}$ day of fermentation.

\section{ZONE OF INHIBITION 14 DAYS}

\section{Samples B.subtilis E.coli S.aureus S.marecens P.aeruginosa}

\begin{tabular}{|c|c|c|c|c|c|}
\hline $\mathrm{BT}+\mathrm{S}$ & --- & $2 \mathrm{~mm}$ & $3 \mathrm{~mm}$ & $5 \mathrm{~mm}$ & --. \\
\hline $\mathrm{BT}+\mathrm{H}$ & --- & --- & --- & $2 \mathrm{~mm}$ & --- \\
\hline$B T+J$ & --- & --- & --- & --- & $4 \mathrm{~mm}$ \\
\hline BT-O & --- & --- & --- & --- & --- \\
\hline $\mathrm{GT}+\mathrm{S}$ & --- & --- & --- & --- & --- \\
\hline $\mathrm{GT}+\mathrm{H}$ & --- & --- & --- & --- & --- \\
\hline$G T+J$ & --- & --- & --- & --- & $5 \mathrm{~mm}$ \\
\hline GT-O & --- & --- & --- & --- & --- \\
\hline S-O & --- & --- & --- & --- & --- \\
\hline J-O & --- & --- & --- & --- & --. \\
\hline $\mathrm{H}-\mathrm{O}$ & --- & --- & --- & --- & --- \\
\hline
\end{tabular}


Table 2 Antimicrobial activity of kombucha samples on $28^{\text {th }}$ day of fermentation.

\begin{tabular}{lccccc}
\hline \multicolumn{5}{c}{ ZONE OF INHIBITION } \\
28 DAYS \\
Samples & B.subtilis & E.coli & S.aureus & S.marecens & P.aeruginosa \\
\hline BT+S & $1 \mathrm{~mm}$ & $4 \mathrm{~mm}$ & $3 \mathrm{~mm}$ & $7 \mathrm{~mm}$ & $3 \mathrm{~mm}$ \\
BT+H & --- & $2 \mathrm{~mm}$ & $2 \mathrm{~mm}$ & $3 \mathrm{~mm}$ & $2 \mathrm{~mm}$ \\
BT+J & $2 \mathrm{~mm}$ & --- & $8 \mathrm{~mm}$ & --- & $6 \mathrm{~mm}$ \\
BT-O & --- & --- & $1 \mathrm{~mm}$ & $1 \mathrm{~mm}$ & $6 \mathrm{~mm}$ \\
GT+S & --- & $2 \mathrm{~mm}$ & $3 \mathrm{~mm}$ & --- & --- \\
GT+H & $1 \mathrm{~mm}$ & $1 \mathrm{~mm}$ & --- & --- & --- \\
GT+J & --- & --- & --- & --- & $5 \mathrm{~mm}$ \\
GT-O & --- & $1 \mathrm{~mm}$ & $3 \mathrm{~mm}$ & $17 \mathrm{~mm}$ & $5 \mathrm{~mm}$ \\
S-O & --- & --- & --- & --- & --- \\
J-O & --- & $9 \mathrm{~mm}$ & $15 \mathrm{~mm}$ & $12 \mathrm{~mm}$ & $3 \mathrm{~mm}$ \\
$\mathrm{H}-\mathrm{O}$ & --- & --- & $5 \mathrm{~mm}$ & $1 \mathrm{~mm}$ & --- \\
\hline
\end{tabular}

Table 3 Antimicrobial activity of kombucha samples on $60^{\text {th }}$ day of fermentation.

\section{ZONE OF INHIBITION \\ 60 DAYS}

\begin{tabular}{lccccc} 
Samples & B.subtilis & E.coli & S.aureus & S.marecens & P.aeruginosa \\
\hline BT+S & $2 \mathrm{~mm}$ & $8 \mathrm{~mm}$ & $5 \mathrm{~mm}$ & $8 \mathrm{~mm}$ & $6 \mathrm{~mm}$ \\
BT+H & --- & $3 \mathrm{~mm}$ & $2 \mathrm{~mm}$ & $4 \mathrm{~mm}$ & $3 \mathrm{~mm}$ \\
BT+J & $3 \mathrm{~mm}$ & $1 \mathrm{~mm}$ & $8 \mathrm{~mm}$ & $1 \mathrm{~mm}$ & $6 \mathrm{~mm}$ \\
BT-O & --- & --- & $2 \mathrm{~mm}$ & $3 \mathrm{~mm}$ & $7 \mathrm{~mm}$ \\
GT+S & $1 \mathrm{~mm}$ & $4 \mathrm{~mm}$ & $3 \mathrm{~mm}$ & $3 \mathrm{~mm}$ & --- \\
GT+H & $1 \mathrm{~mm}$ & $1 \mathrm{~mm}$ & --- & --- & --- \\
GT+J & $4 \mathrm{~mm}$ & $6 \mathrm{~mm}$ & $5 \mathrm{~mm}$ & $3 \mathrm{~mm}$ & $5 \mathrm{~mm}$ \\
GT-O & $1 \mathrm{~mm}$ & $6 \mathrm{~mm}$ & $3 \mathrm{~mm}$ & $18 \mathrm{~mm}$ & $6 \mathrm{~mm}$ \\
S-O & $1 \mathrm{~mm}$ & $1 \mathrm{~mm}$ & $1 \mathrm{~mm}$ & $1 \mathrm{~mm}$ & $1 \mathrm{~mm}$ \\
J-O & --- & $9 \mathrm{~mm}$ & $17 \mathrm{~mm}$ & $16 \mathrm{~mm}$ & $3 \mathrm{~mm}$ \\
$\mathrm{H}-\mathrm{O}$ & $2 \mathrm{~mm}$ & $1 \mathrm{~mm}$ & $5 \mathrm{~mm}$ & $1 \mathrm{~mm}$ & --- \\
\hline
\end{tabular}

\section{CONCLUSION}

The study undertaken showed that the antioxidant and antimicrobial activities of kombucha with prolonged fermentation time of 60 days and with change in carbon source were observed. From the study, sample containing green tea and white sugar $(\mathrm{GT}+\mathrm{S})$ was found to be the most antioxidant on 7 th day of fermentation by showing about $84 \%$ of DPPH inhibition. However, fermentation could reduce the antioxidant properties of the samples after 7th day of fermentation. For antimicrobial properties, black tea with white sugar $(\mathrm{BT}+\mathrm{S})$ was the best substrates. It showed inhibition against the microbes from 14th day of fermentation and it was further increased till the 60th day that effectively inhibited all five strains of bacteria. The use of jaggery as carbon source showed interesting observation that needed further investigation to understand its roles in changing the antioxidant and antimicrobial properties of kombucha. Our results proved that changes in fermentation time and media could affect the antioxidant and antimicrobial efficacies of kombucha. This study could help in choosing the best substrate for preparation of kombucha according to the therapeutic effects desired. This could also be guideline for further investigations regarding the understanding of biochemical changes in fermentation of kombucha.

\section{ACKNOWLEDGEMENT}

This research was funded by GUP-2017-053 that granted by Universiti Kebangsaan Malaysia and the Ministry of Higher Education Malaysia.

\section{REFERENCES}

Bhattacharya, D., Bhattacharya, S., Patra, M. M., Chakravorty, S., Sarkar, S., Chakraborty, W., Koley H., Gachhui, R. 2016. Antibacterial activity of polyphenolic fraction of kombucha against enteric bacterial pathogens. Current Microbiology, 73, 885-896.

Bhattacharya, D., Ghosh, D., Bhattacharya, S., Srakar, S., Karmakar, P., Koley, H., Gachhui, R. 2017. Antibacterial activity of polyphenolic fraction of Kombucha against Vibrio cholerae: targeting cell membrane. Letters in Applied Microbiology, 66, 145-152.

Cetojevic-Simin, D. D., Bogdanovic, G. M., Cvetkovic, D. D., Velicanski, A. S. 2008. Antiproliferative and antimicrobial activity of traditional Kombucha and Satureja montana L. Kombucha. Journal of BUON, 13, 395401.

Chen, C., Liu, B. Y. 2000. Changes in major components of tea fungus metabolites during prolonged fermentation. Journal of Applied Microbiology, 89, 834-839.

Chou, C. C., Lin, L. L., Chung, K. T. 1999. Antimicrobial activity of tea as affected by the degree of fermentation and manufacturing season. International Journal of Food Microbiology, 48, 125-130.

Chu, S. C., Chen, C. 2006. Effects of origins and fermentation time on the antioxidant activities of kombucha. Food Chemistry, 98, 502-507.

Dufresne, C., Farnworth, E. 2000. Tea, Kombucha, and health: A review, 33.

Fatima, A., Malik, F., Shafiq, A., Jawaid, S., Hakim, S. T., Nadeem, S. G. 2016. Evaluation antibacterial activity of three most consumed tea extracts against pathogenic bacteria. International Journal of Current Microbiology and Applied Science, 5, 824-827.

Fessenden, R. J., Fessenden, S. 1994. Organic Chemistry. Belmont, CA Brooks/Cole Publishing (pp. 246-260).

Fu, C., Yan, F., Cao, Z., Xie, F., Lin, J. 2014. Antioxidant activities of Kombucha prepared from three different substrates and changes in content of probiotics during storage, Journal of Food Science and Technology, 34, 123-126.

Gramza-Michalowska, A., Kulczynski, B., Xindi, Y., Gumienna, M. 2016. Research on the effect of culture time on the kombucha tea beverage's antiradical capacity and sensory value. Acta Scientiarum Polonorum Technologia Alimentaria, 15, 447-457.

Greenwalt, C., Ledford, R., Steinkraus, K. 1998. Determination and characterization of the antimicrobial activity of the fermented tea Kombucha. LWT - Journal of Food Science and Technology, 31, 291-296.

Greenwalt, C. J., Steinkraus, K. H., Ledford, R. 2000. Kombucha, the fermented tea: Microbiology, composition, and claimed health effects. Journal of Food Protection, 63, 976-981.

Hesseltine, C. W., 1965. A millenium of fungi, food and fermentation. Mycologia, 57, 149-197.

Huang, D., Ou, B. and Prior, R. L. 2005. The chemistry behind dietary antioxidant capacity assays. Journal of Agricultural and Food Chemistry, 53(6), 1841-1856

Jayabalan, R., Marimuthu, S., Swaminathan, K. 2006. Changes in content of organic acids and tea polyphenols during kombucha tea fermentation. Food Chemistry, 102, 392-398.

Jayabalan, R., Subathradevi, P., Marimuthu, S., Sathishkumar, M. and Swaminathan, K. 2008. Changes in free-radical scavenging ability of kombucha tea during fermentation. Food Chemistry, 109, 227-234

Lobo., R. O., Dias, F. O., Shenoy, C. K. 2017. Kombucha for healthy living: evaluation of antioxidant potential and bioactive compounds. International Food Research Journal, 24, 541-546.

Mo, H., Zhu, Y., Chen, Z. 2008. Microbial fermented tea - a potential source of natural food preservatives. Trends in Food Science and Technology, 19 $124-130$.

Sharma, O. P., Bhat, T. K., 2009. DPPH antioxidant assay revisited. Food Chemistry, 113, 1202-1205.

Sievers, M., Lanini, C., Weber, A., Schuler-Schmid, U., Teuber, M. 1995. Microbiology and fermentation balance in a Kombucha beverage obtained from a tea fungus fermentation. Systematic and Applied Microbiology, 18, 590-594.

Sreeramulu, G., Zhu, Y., Knol, W. 2000. Kombucha fermentation and its antimicrobial activity. Journal of Agricultural and Food Chemistry, 48, 2589-2594.

Srihari, T., Karthikesan, K., Ashokkumar, N., Satyanarayana, U. 2013 Antihyperglycaemic efficacy of kombucha in streptozotocin-induced rats. Journal of Functional Foods, 5, 1794-1802. 
Sumbhate, S. V., Nayak, S., Goupale, D., Tiwari, A., Jadon, R. S. 2012

Colorimetric Method for the estimation of ethanol in alcoholic-drinks. Journal of Analytical Techniques, 1, 1-6.

Velićanski, A. S., Cvetković, D. D., Markov, S. L., Tumbas V. T., Šaponjac, Vulić, J. J. 2014. Antioxidant and antibacterial activity of the beverage obtained by fermentation of sweetened lemon balm Melissa officinalis L. tea with symbiotic consortium of bacteria and yeasts. Food Technology and Biotechnology, 52, 420-429.

Vīna, I., Semjonovs, P., Linde, R., Denina, I. 2014. Current evidence on physiological activity and expected health effects of Kombucha fermented beverage. Journal of Medicinal Food, 17, 179-188.

Yang, Z., Ji, B., Zhou, F., Li, B., Luo, Y., Yang, L., Li, T. 2008 Hypocholesterolaemic and antioxidant effects of kombucha tea in high cholesterol fed mice. Journal of the Science of Food and Agriculture, 89, $150-156$. 